\title{
CARACTERIZACIÓN Y CLASIFICACIÓN GEOQUÍMICA DE ASFALTITAS CUBANAS
}

\author{
Zulema Dominguez \\ COPPE/UFRJ - Instituto Alberto Luiz Coimbra de Pós-graduação e Pesquisa em Engenharia, Universidade Federal de Rio de \\ Janeiro, Rio de Janeiro - RJ, Brasil / CEINPET/CUPET -Centro de Investigaciones del Petróleo, Habana, Cuba \\ Jarbas Vicente Poley Guzzo \\ Petrobras/CENPES/PDEXP/GEOQ, Ilha do Fundão, 21941-901 Rio de Janeiro - RJ, Brasil \\ Débora de Almeida Azevedo* \\ Instituto de Química, Universidade Federal de Rio de Janeiro, 21941-909 Rio de Janeiro - RJ, Brasil \\ Recebido em 11/5/07; aceito em 7/3/08; publicado na web em 4/9/08

\begin{abstract}
GEOCHEMICAL CHARACTERIZATION AND CLASSIFICATION OF CUBAN ASPHALTITES. Traditional biomarker parameters and aromatic compounds were applied to characterize and classify ten Cuban asphaltites (asphaltene-rich petroleum occurring as seeps or filling veins, joints, cavities and fissures). Genetic molecular parameters were compared in order to establish oil-oil correlations between samples. Thermal evolution was investigated using saturated biomarker and aromatic maturity parameters. All samples seem to represent petroleum in the early catagenetic stage. Statistical procedures used as auxiliary techniques show that they represent oils siliciclastic suboxic sourced oils).
\end{abstract} \\ of Family II (marine anoxic carbonate sourced oils), except for 2 samples interpreted as belonging to Family III oils (normal marine
}

Keywords: biomarker; biodegradation; statistical analysis.

\section{INTRODUCCIÓN}

En la exploración y desarrollo de un campo petrolero, los geólogos, ingenieros y geofísicos plantean sus objetivos en términos de configuración estructural de la roca o disminuciones de presión. Los geoquímicos ubican los fluidos de los yacimientos e integran la data geoquímica dentro de la red geológica y la historia de producción del campo, enfocando su interés en la determinación de los tipos de petróleo presentes en un área e intentando predecir el número y características de las rocas madres.

La geoquímica orgánica estudia la generación, migración y acumulación del petróleo bajo la superficie; estableciendo límites entre facies potenciales como rocas madres y las facies orgánicas que se relacionan estratigráficamente; además define las profundidades a las cuales prevalecieron las condiciones diagenéticas óptimas para la formación del petróleo.

Esto significa que el geoquímico define cómo se formó el petróleo, dónde está localizado, a qué profundidad y cuál es su patrón de migración desde la roca madre hasta las facies porosas del yacimiento.

Los estudios de geoquímica orgánica realizados en Cuba hasta el momento son bastante incipientes e incluyen: la caracterización de los principales yacimientos de petróleos, estudios de rocas madre, determinación de las tendencias de la maduración y los gradientes geotérmicos; y en los últimos tiempos, a partir de una integración de todos los estudios anteriores, la identificación y evaluación de los principales sistemas petroleros conocidos hasta el momento.

\section{Caracterización de los petróleos}

Desde el punto de vista exploratorio los petróleos se caracterizan genética y comercialmente. La primera caracterización se refiere al origen de los crudos, es decir a las particularidades de las rocas que

*e-mail: debora@iq.ufrj.br los generaron; mientras que la segunda se realiza fundamentalmente a partir de dos parámetros: densidad (expresada en ${ }^{\circ} \mathrm{API}$ ) y contenido de azufre, aunque también son tomados en cuenta otros parámetros como la viscosidad y la acidez.

\section{Pertenencia genética}

En términos de caracterización genética los petróleos son clasificados en familias, que van a agrupar a los crudos generados por una misma roca madre; los cuales van a tener características semejantes entre sí, aspecto que se refleja en los biomarcadores. En Cuba se han descrito tres familias genéticas de petróleo. ${ }^{1}$ Familia I: Hidrocarburos generados por rocas madre carbonatadas con materia orgánica principalmente marina (tipo II, IIs); depositadas en ambiente fuertemente reductor (anóxico, hipersalino). Familia II: Hidrocarburos generados por rocas madre carbonatadas a partir de materia orgánica tipo II, fundamentalmente marina depositada en ambiente reductor, menos anóxico que la família I. Familia III: Hidrocarburos generados por rocas madre siliciclásticas carbonatadas con materia orgánica marina con aporte terrestre, tipo II (II-III), depositadas en condiciones subóxicas.

\section{Calidad comercial}

El conocimiento de las calidades comercial de los petróleos presentes en determinada área, sector o bloque es de vital importancia para disminuir los riesgos en el proceso de exploración, debido a que una acumulación de petróleo sobre la base de la cantidad de reservas in situ y sobre todo de la calidad del hidrocarburo que almacena, puede constituir un yacimiento ó no. Es por ello que conocer y estudiar los procesos que afectan las propiedades físico - químicas del petróleo, constituye una tarea vital de la geoquímica de exploración. Los principales procesos que determinan la calidad comercial de los crudos se pueden agrupar en dos grandes grupos: procesos primarios - origen; procesos secundarios - maduración, biodegradación, lavado por agua, fraccionamiento evaporativo y desasfaltinización. 


\section{Procesos primarios}

Son los procesos que determinan las calidades comerciales del petróleo desde el mismo momento de su generación y están determinados por las características orgánicas y térmicas de las rocas madre, es decir ocurren a nivel de roca madre.

\section{Procesos secundarios}

La composición final del petróleo puede ser fuertemente influenciada por alteraciones que ocurren durante la migración secundaria y después de la acumulación. La alteración del petróleo tiende a causar modificaciones en sus características, influenciando en su calidad y valor económico, afectando además desfavorablemente los estudios geoquímicos. Estos procesos ocurren a nivel de reservorio.

\section{Sistema petrolero}

La relevancia de la geoquímica prevalece, a partir de que la existencia del petróleo resulta de la interacción de procesos físicos y químicos controlados por la historia geológica de una cuenca. Esta comprensión condujo a finales de la década del 70 al concepto "Sistema Petrolero", el cual enfatiza la historia dinámica de procesos geológicos y eventos. Los procesos químicos al comienzo de la cadena de eventos llevan a la existencia de petróleo: la deposición de horizontes generadores y transformaciones termo-cinéticas de la materia orgánica preservada conllevan a la formación de petróleos. Los procesos físicos controlan los procesos de migración y entrampamiento. El tiempo correcto de los eventos es la esencia para el desarrollo de acumulaciones de petróleo comercial. Hoy se acepta en la industria del petróleo que la aplicación de la geoquímica orgánica incrementa significativamente las posibilidades de éxito en la exploración petrolera.

El sistema petrolero, se ha convertido en la herramienta operacional moderna escogida para la evaluación cuantitativa de los riesgos en la exploración petrolera. Como es conocido el concepto de sistemas petroleros encierra una roca madre activa y todas las manifestaciones de petróleo (comerciales o no) relacionadas genéticamente con ella. Por ende el punto de partida para la aplicación del método es la clasificación genética de todas las manifestaciones de petróleo conocidas en el área en cuestión. Esta tarea es de carácter sistemático, ya que sobre la base de la cantidad de manifestaciones de petróleo clasificadas genéticamente que se tenga en determinada cuenca, área o sector; mejor definidos estarán los aspectos temporales (edad, momento crítico) y espaciales (límite y extensión geográfica, extensión estratigráfica) de los sistemas petroleros presentes. En el caso particular de Cuba, el grado de enmascaramiento que presentan estos sistemas ocasiona que en algunos casos no se conozcan algunos de sus elementos y no sea posible definir otros con toda seguridad, ${ }^{2}$ razón esta por la que los compuestos biomarcadores se han convertido en una herramienta poderosa para la caracterización geoquímica.

\section{Biomarcadores}

Los biomarcadores son moléculas orgánicas complejas compuestas de carbono, hidrógeno y otros elementos. Estas moléculas sobreviven a los procesos de sedimentación y enterramiento de la materia orgánica con poco o ningún cambio en su estructura respecto a las moléculas precursoras en organismos vivos. Estes compostos aportan mucha información en el estudio de los petróleos, ya que permiten la determinación de la fuente biológica de la materia orgánica, el paleoambiente de depositación, la madurez de la materia orgánica, entre otros parámetros. Estos compuestos también se conocen como "fósiles geoquímicos" o "marcadores biológicos". 3,4

En el presente estudio fueron empleados parámetros de bio- marcadores tradicionales derivados de los esteranos y terpanos así como algunos parámetros de aromáticos logrando caracterizar y clasificar diez muestras de asfaltitas: muestras de petróleo ricas en asfaltenos, las que pueden ser encontradas como manifestaciones superficiales, llenando venas, cavidades y fisuras de rocas en superficie. Las muestras colectadas corresponden a localidades ubicadas en el Occidente y Oriente de Cuba. Estas muestras se encuentran afectadas por biodegradación, en algunos casos hasta los esteranos y algunos compuestos aromáticos de bajo peso molecular. Dadas las características de las muestras en estudio se puso especial interés en establecer correlaciones que envolvieran preferencialmente a compuestos biomarcadores tricíclicos y tetracíclicos ${ }^{5,6}$ por la mayor estabilidad de estos compuestos ante procesos de maduración y biodegradación, así como el estudio de compuestos aromáticos, también conocidos por su resistencia a alteración por biodegradación y útiles en la determinación de correlaciones con respecto a contribución de materia orgánica, regímenes originales de deposición y grado de evolución térmica de la matéria orgánica. ${ }^{3,4,7-11}$ En la Tabla 1 se muestran los valores de los parámetros moleculares determinados para realizar la clasificación.

Estudios anteriores, ${ }^{1}$ han permitido clasificar los petróleos cubanos en tres familias fundamentales. El objetivo fundamental de este estudio consistió en definir la pertenencia genética de los extractos estudiados a las familias conocidas y correlacionar con los sistemas petroleros cubanos ya definidos. ${ }^{2}$

\section{EXPERIMENTAL}

Se colectaron 10 muestras de asfaltitas correspondientes a varias localidades del territorio cubano, las cuales fueron subdivididas en ocho manifestaciones superficiales, (LR, CB, MO, LB1, AM2, FS, $\mathrm{SMB}$ y M) y dos muestras de asfaltos (CC y ME). Los extractos fueron obtenidos mediante extracción por soxhlet, con diclorometano como solvente. La separación de los extractos en fracciones fue mediante equipamiento Aspec XL (Wilson), se utilizó cartucho para extracción en fase sólida de sílica gel - $1000 \mathrm{mg} / 6 \mathrm{~mL}$ de capacidad (Applied Separations). Se pesó cerca de $150 \mathrm{mg}$ de muestra en un frasco de vidrio de $7 \mathrm{~mL}$ de capacidad, se adicionó $3,0 \mathrm{~mL}$ de $n$-hexano y se colocó la muestra en equipo de ultrasonido por algunos segundos para auxiliar en la homogenización. Fueron inyectados $1,5 \mathrm{~mL}$ de la solución en el equipamiento, previamente acondicionado con $n$-hexano. La elusión de los compuestos saturados y aromáticos se realizó con $18 \mathrm{~mL}$ de $n$-hexano ( 6 corridas de 3,0 mL). Las fracciones de compuestos saturados y aromáticos fueron evaporadas con flujo de nitrógeno. Una vez las fracciones secas se diluyeron en volumen conocido de $n$-hexano e inyectaron en equipamiento cromatográfico, el cartucho utilizado fue descartado.

La separación cromatográfica fue realizada mediante cromatografía líquida de alta resolución, (High Performance Liquid Chromatography, HPLC), en cromatografo líquido Waters con controlador, bombas, auto muestreador, detectores de índice de refracción y ultravioleta $(\lambda, 254$ y $270 \mathrm{~nm})$ además de dos columnas semipreparativas en serie de 10 × $250 \mathrm{~mm}$ (Waters Spherisorb $S 5 \mathrm{~W}$ - Part Number: PSS830185). Una vez realizada la separación, la fracción de saturados, fue entonces analizada por cromatografía gaseosa en equipamiento 5890A de la Agilent Technologies USA, con inyector split/splitless y columna capilar de alta resolución (DB5). La identificación de los compuestos de interés fue realizada mediante cromatografía gaseosa acoplada a espectrometría de masas (CG-EM) en equipamiento 5890/II (CG) con interfase para 5870MSD (EM) de Agilent Technologies. El experimento de monitoreo selectivo de iones se realizó monitoreando los iones $\mathrm{m} / \mathrm{z}$ 191, 177, 217, 218 y 259 para los compuestos saturados y en la fracción de aromáticos los iones 
Tabla 1. Principales parámetros geoquímicos analizados

\begin{tabular}{lcccccccccc}
\hline Muestras & LR & CB & MO & CC & ME & LB1 & AM2 & FS & SMB & M \\
\hline C23-3/C21-3 & 2,92 & 3,75 & 1,96 & 2,25 & 2,92 & 3,38 & 2,23 & 3 & 2,34 & 2,96 \\
H29/H30 & 0,86 & 0,51 & 1,68 & 1,16 & 1,55 & 0,91 & 0,87 & 4,57 & 0,94 & 0,75 \\
H35/H34 & 1,35 & 0,62 & 0,89 & 1,08 & 1,1 & 1,22 & 1,06 & 0,9 & 0,98 & 0,72 \\
TET24/26Tri & 2 & 0,63 & 8,16 & 2,75 & 5,74 & 2,43 & 4,47 & 1,1 & 1,16 & 0,71 \\
TET 24/H30 & 0,15 & 0,04 & 0,38 & 0,12 & 0,15 & 0,08 & 0,1 & 0,18 & 0,13 & 0,18 \\
TPP & 0,44 & 0,84 & 0,78 & 0,52 & 0,48 & 0,41 & 0,09 & 0,46 & 0,42 & 0,77 \\
G/H30 & 0 & 0,02 & 0.07 & 0,26 & 0,14 & 0,1 & 0,13 & 0,09 & 0,3 & 0,03 \\
Diast/Est. reg & 0,78 & 0,34 & 0,06 & 0,4 & 0,17 & 0,19 & 0,22 & 0,8 & 0,84 & n.d \\
\%C29/C27 & 1.52 & 1.57 & 0,46 & 1,12 & 1,05 & 1 & 1,91 & 2,35 & 0,96 & n.d \\
Hop/Est & 0,65 & 8,52 & 8,76 & 7,37 & 5,73 & 3,36 & 0,6 & 2,31 & 3,08 & n.d \\
Ts/Tm & 0,33 & 0,98 & 0,94 & 0,26 & 0,23 & 0,15 & 0,18 & 0,6 & 0,72 & 0,8 \\
PP- 1 & 1,74 & 0,94 & 1,22 & 1,08 & 1,93 & 1,37 & 1,42 & 0,91 & 2,71 & 1,19 \\
MFI-1 & 0,57 & 1 & 0,82 & 0,93 & 0,52 & 0,73 & 0,7 & 1,1 & 0,37 & 0,84 \\
RMFI & 0,85 & 1 & 0,93 & 0,96 & 0,84 & 0,9 & 0,89 & 1,01 & 0,79 & 0,93 \\
DBT/F & 2,6 & 0,3 & 1,21 & 2,62 & 2,5 & 0,32 & 0,25 & 0,05 & 0,36 & 0,27 \\
DBT/MDBT & 0,26 & 0,12 & 0,06 & 0,34 & 0,08 & 0,12 & 0,12 & 0,65 & 0,03 & 0,33 \\
MDR & 1,02 & 2,2 & 1,82 & 2,62 & 1,46 & 1,28 & 0,66 & 0,94 & 1,03 & 2,96 \\
F/(F+MF) & 0,2 & 0,13 & 0,1 & 0,38 & 0,08 & 0,21 & 0,31 & 0,6 & 0,1 & 0,4 \\
\hline PP-1 (1MF+9 & & 0,29 &
\end{tabular}

$\mathrm{PP}-1=(1 \mathrm{MF}+9 \mathrm{MF}) /(2 \mathrm{MF}+3 \mathrm{MF}), \mathrm{MFI} 1=1.89(2 \mathrm{MF}+3 \mathrm{MF}) / \mathrm{F}+1.26(1 \mathrm{MF}+9 \mathrm{MF}), \mathrm{MDR}=4 \mathrm{MDBT} / 1 \mathrm{MDBT} . \mathrm{RMFI}=0.3 *(\mathrm{MFI}-1)+0.68, \mathrm{TPP}=\mathrm{Po}-$ liprenóides tetracíclicos/ C27 diast. Tm: C27 17 $\alpha(\mathrm{H})-22$, 29, 30- trisnorhopano, Ts: C27 18 $\alpha(\mathrm{H})-22$, 29, 30-trisnorneohopano, G: Gamacerano

$m / z, 178,192,184,198$ y 206.

El procesamiento estadístico de los datos fue realizado mediante técnicas de estadística multivariada, utilizando análisis de componentes principales y análisis Cluster, el procesamiento fue realizado mediante el empleo del sofware Statistic 7.0.

\section{RESULTADOS Y DISCUSIÓN}

Las muestras analizadas se caracterizan por mostrar presencia de biodegradación con diferentes grados de extensión. La cromatografía gaseosa de los petróleos totales cuando presente, muestra ausencia de $n$-parafinas e isoprenoides pristano y fitano, así como gibas de compuestos no resueltos más pronunciadas hacia los finales pesados (zona de biomarcadores), esto es observado en 4 de las 10 muestras: CB, SMB, M y LR. En 3 muestras no fue posible obtener el cromatograma de gases. Estas fueron ME, LB1, AM2 y ocurrió un detalle curioso. En las 3 muestras restantes se muestra evidencia de mas de un pulso de generación, pues se muestra un perfil, con los finales biodegradados y un pulso preservado de parafinas de bajo peso molecular. Este es el caso de las muestras FS, CC y MO, en las dos últimas se observa que este segundo pulso aun no ha sido biodegradado (pristano y fitano, se encuentran en menor proporción que las parafinas lineares correspondientes, dígase $n$-C17 y $n$-C18).

Los cromatogramas de masas, correspondientes a los hopanos $(\mathrm{m} / \mathrm{z}, 191)$ y esteranos $(\mathrm{m} / \mathrm{z} 217)$, representados en la Figura $2 \mathrm{~S}$, muestran el predominio de medio carbonático entre las muestras lo que se evidencia por la razón $\mathrm{H}_{29} / \mathrm{H}_{30}>1$ y $\mathrm{H}_{35} / \mathrm{H}_{34}>1$ fundamentalmente. No fue posible identificar los esteranos en la muestra $\mathrm{M}$, el cromatograma de masas correspondiente muestra sólo un perfil de compuestos no resueltos, de las restantes muestras sólo 2 muestran cantidades significativas de esteranos estás son AM2 y LB1; aun así, fue posible cuantificar los esteranos $\mathrm{C}_{27}, \mathrm{C}_{28}$ y $\mathrm{C}_{29}$ así como las relaciones $[20 \mathrm{~S} /(20 \mathrm{~S}+20 \mathrm{R})]$ y $[\alpha \beta \beta /(\alpha \beta \beta+\alpha \alpha \alpha)]$ en las nueve muestras restantes.

La serie de los 25 norhopanos apareció en 8 de las 10 muestras, siendo elevada en 4 de las muestras con valores de la razón 25Nor/Hop entre (0.4-0.84) en la muestra $\mathrm{M}$ no fue posible cuantificar el valor y las muestras LB1, MO y M.E exhiben valores muy bajos $(<0.05)$, lo que sugiere la degradación de las mismas en superficie, producto de evaporación de los compuestos mas ligeros, Tabla 1.

El estudio de compuestos aromáticos en las muestras permitió comprobar el predominio de Dibenzotiofeno (DBT) sobre Fenantreno (F), así como de Metildibenzotiofeno (MDBT), sobre Metilfenantreno (MF) en 4 de las muestras estudiadas (LR, MO, CC, y M.E), demostrando el carácter mas carbonático de estas muestras. ${ }^{12} \mathrm{El}$ patrón de distribución de los MDBT (4MDBT>2+3MDBT<1MDBT), que segun Hugues ${ }^{12}$ indicaría medio carbonático, se cumple para las muestras de LR, LB1, AM2 y SMB, aunque este parámetro según Schou y Myhr, ${ }^{13}$ sugiere mas bien el carácter poco evolucionado de las muestras, lo cual se corresponde con el estudio realizado con los parámetros de evolución térmica determinados en el presente estudio. El otro patrón seguido por las muestras es de 4MDBT $>2+3 \mathrm{MDBT}>1 \mathrm{MDBT}$, este patrón es seguido por las muestras $\mathrm{CB}, \mathrm{MO}, \mathrm{ME}$ y M, este tipo de patrón se ha identificado en algunos casos con muestras depositadas en rochas de aporte siliciclástico, ${ }^{12}$ Figura 3S. Las dos muestras restantes CC y FS muestran predominio de 2+3MDBT, lo que podría indicar la mayor resistencia de este compuesto a la biodegradación, producto de que la velocidad de la biodegradación en los compuestos aromáticos decrece con el incremento de los anillos y con el incremento de alquilsustituyentes, siendo que la velocidad de esta secuencia de biodegradación es influenciada por la posición del sustituyente alquilo. ${ }^{14}$

Debe decirse que aunque fue posible medir las relaciones de compuestos aromáticos las cantidades relativas de fenantreno y sus compuestos alquilados están en bajas proporciones, sobre todo en las muestras de CC, MO, ME, SMB y M. No fue posible de medir en ninguno de los casos las cantidades de naftaleno y sus derivados alquilados, probablemente por el mayor grado de volatilidad de este compuesto, además del origen de las muestras. El bibenzotiofeno y sus derivados alquilados, se presentan como el grupo de compuestos aromáticos predominante en el conjunto de muestras.

Para el estudio de evolución térmica se utilizaron tanto relaciones 
de compuestos saturados como de aromáticos. Para los compuestos saturados fueron medidos los isómeros de $\mathrm{C}_{29}$ esteranos [20S/(20S+20R) y $\alpha \beta \beta /(\alpha \beta \beta+\alpha \alpha \alpha)$ ], comúnmente empleados en la determinación de madurez, Figura 1a. No fue posible medir las relaciones para el caso de la muestra M, producto de la imposibilidad de cuantificación de esteranos en esta muestra.

En ninguna de las muestras restantes, donde fueron calculadas estas relaciones, se alcanza el equilibrio mostrándose las muestras de MO, CB, M.E como las mas maduras dentro del conjunto, Figura 1.

Las relaciones Ts/Tm presentan valores entre 0.15 y 0.98 . Es sabido que esta relación se incrementa con la evolución térmica producto de la mayor estabilidad térmica del isómero Ts, pero también es conocido que esta razón es afectada por la variación de facies, por lo que los resultados de este parámetro deben ser analizados con extremo cuidado, cuando se trabaja con muestras de diferente origen. Los mayores valores alcanzados para este parámetro corresponden a las muestras CB, MO, y ME, Figura 1b. La relación entre 23/21 tricíclicos, también ha sido propuesta como parámetro indicador de evolución térmica ${ }^{15} \mathrm{y}$ fue usada en el presente estudio, por el hecho que estos compuestos presentan mayor resistencia al proceso de biodegradación. En base a este parámetro se presentan como las muestras más maduras CB, LB1 y FS, Tabla 1, no puede decirse que exista mucha consistencia entre los resultados obtenidos en las dos
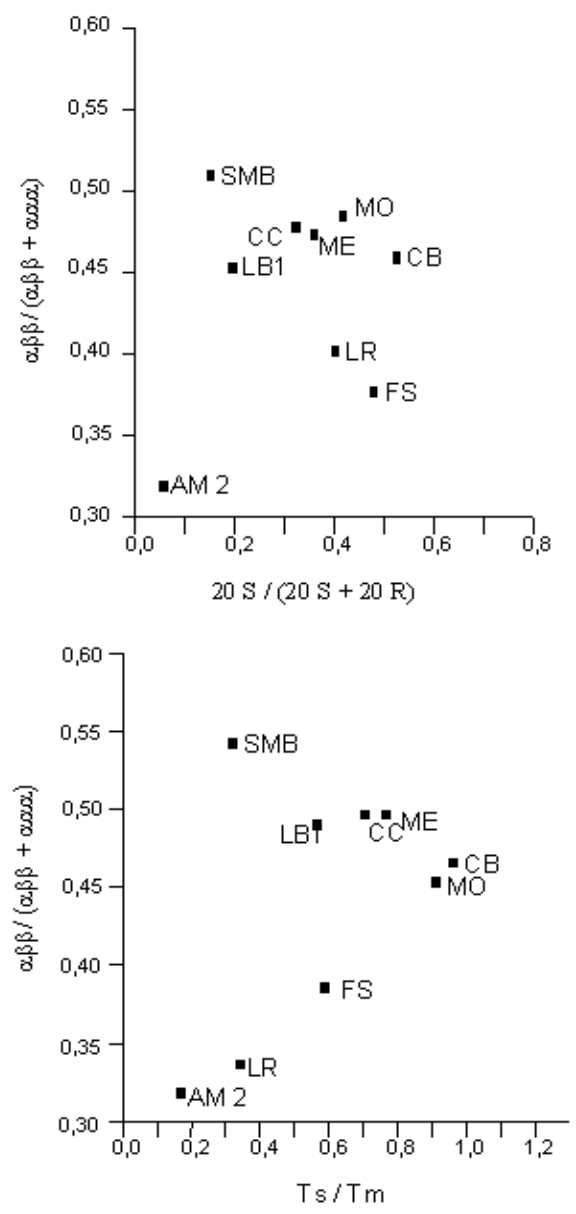

Figura 1. Parámetros indicadores de evolución térmica en esteranos (20S/20S+20R), basada en la isomerización de las posiciones $C_{14}$ e $C_{17}$ para los compuestos 20R e 20S de esteranos regulares y $\alpha \beta \beta /(\alpha \beta \beta+\alpha \alpha \alpha))$ o sea: $5 \alpha(H), 14 \beta(H), 17 \beta(H) /[(5 \alpha(H), 14 \beta(H), 17 \beta(H))+(5 \alpha(H), 14 \alpha(H), 17 \alpha(H)] y$ [Ts/Tm vs $\alpha \beta \beta /(\alpha \beta \beta+\alpha \alpha \alpha)]$. Estructuras químicas en Figura 3 variantes a no ser por la muestra $\mathrm{CB}$. Estas diferencias pueden estar influenciadas por la presencia de variación de facie ${ }^{16} \mathrm{y}$ en menor medida de variación de salinidad entre las muestras. ${ }^{17}$

También fue medida la evolución térmica de las muestras utilizando relaciones de compuestos aromáticos características para este tipo de estudio. Fueron utilizados el fenantreno y el metilfenantreno, isómero metilado del fenantreno, para calcular el índice de metil fenantreno (MFI-1) y el índice de madurez (PP-1). ${ }^{10,11}$ También fue calculado el parámetro de evolución térmica MDR y DBT/F. ${ }^{11,18}$ Los valores de estos parámetros pueden ser observados en la Tabla 1.

Del estudio realizado con los compuestos aromáticos se llegó a que todas las muestras se encuentran en la etapa de catagénesis temprana, mostrándose dentro del conjunto como las más maduras desde el punto de vista del cálculo de PP-1, las muestras de CB y FS y desde el punto de vista del cálculo de MDR las muestras M, CB y CC las que podrían ubicarse en el comienzo de la ventana de generación de petróleo, ${ }^{19}$ aunque cabe destacar que la muestra de CC es un asfalto, por tanto rica en compuestos sulfurados, por tanto no debe ser comparadas con el resto de las muestras superficiales.

\section{Procesamiento estadístico de los datos geoquímicos}

El análisis exploratorio de datos multivariados se ha mostrado de gran utilidad en el análisis geoquímico para la exploración - producción que necesita cada vez más de respuestas más rápidas. Mediante la quimiometría, es posible decidir que determinaciones son mas importantes, de esta forma pudiendo suprimir las de menor importancia a través del análisis de componentes principales. ${ }^{20}$ También es posible hacer agrupamientos por similitud, representando esta información en forma de un diagrama bidimensional llamado de dendograma, realizando esto a través del análisis por agrupamiento jerárquico. ${ }^{21}$

En el presente estudio, fueron aplicadas las dos técnicas, con el objetivo de observar el comportamiento geoquímico descrito por las muestras. Fue utilizado el sofware Statistica 7.0. Para la obtención de los dendogramas fueron utilizados la distancia euclidiana y el método de conexión por el vecino mas distante (Complete Linkage o Furthest Neighbor), con el propósito de encontrar la mayor similitud entre las muestras del conjunto. ${ }^{22}$

Fue realizado el análisis de componentes principales, siendo la Figura 2a la representación de los Scores de PC1 vs PC2, evidenciándose el agrupamiento de las muestras segun sus características. En este caso son separadas del conjunto, dos muestras, las correspondientes a CB y M. Por su parte el análisis cluster, mostró que el conjunto de muestras fue subdividido en 4 miniconjuntos, el primero compuesto por 6 muestras, correspondientes a muestras superficiales, un segundo constituido por la muestra $\mathrm{CB}$, un tercer conjunto constituido por las dos muestras de asfaltos y un último conjunto constituido por la muestra de M, la que presenta la mayor afectación por biodegradación, que llega hasta los esteranos. La distribución de las muestras puede observarse en la Figura 2b.

El primer conjunto constituido por las muestras de superficie, fue ubicado como perteneciente a la familia II de petróleos cubanos, el segundo conjunto constituido por la muestra de CB, con características de las famílias II y III de petróleos cubanos, el tercer conjunto constituido por las muestras de asfaltos, ubicados en el presente estudio como perteneciente a la familia II de petróleos cubanos y el último conjunto, el mas separado, constituido por M la muestra con las señales mas fuertes de biodegradación, afectado hasta los esteranos e con características correspondientes a las famílias II y III de petróleos cubanos. Resulta interesante el hecho que tanto el análisis de componentes principales, como el análisis cluster apuntan hacia el mismo agrupamiento, separandose en ambos análisis las muestras $\mathrm{M}$ y $\mathrm{CB}$, reafirmando la coherencia de los resultados. 
a)

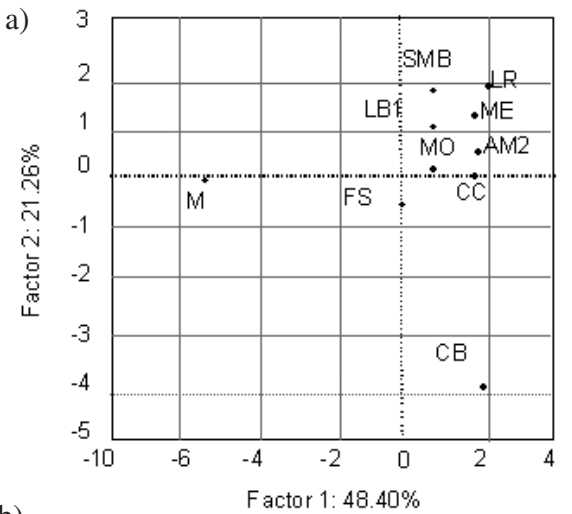

b)

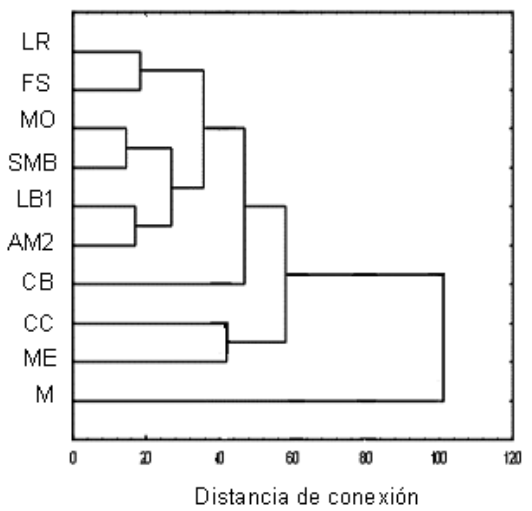

Figura 2. Análisis estadístico: (A) Scores de componentes principales (PCA); (B) Cluster de las muestras
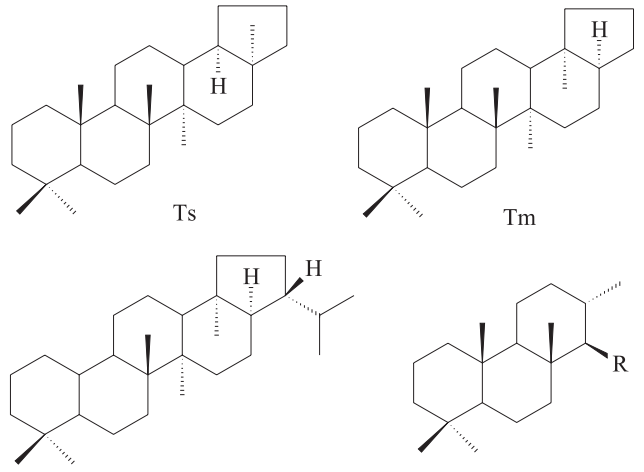

25-Nor-17 $\alpha(\mathrm{H}), 21 \beta(\mathrm{H})$-Hopano

Terpano Tricíclico
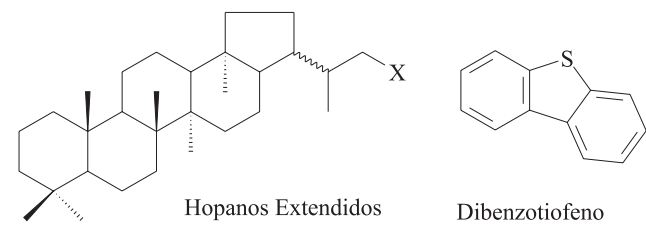

Dibenzotiofeno
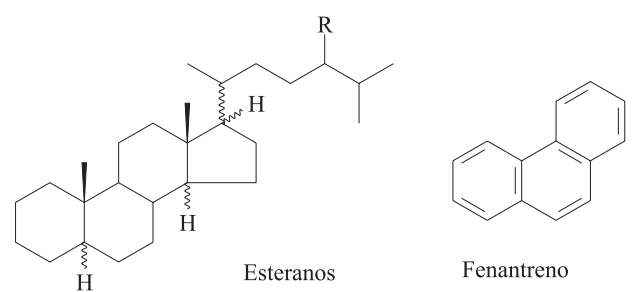

Figura 3. Estructuras químicas de los principales compuestos presentados en el estudio

\section{CONCLUSIONES}

De los resultados obtenidos usando las relaciones de biomarcadores en la fracción de compuestos alifáticos, así como la utilización de parámetros de geoquímicos de la fracción de compuestos aromáticos y el análisis de estadística multivariada nos permiten concluir que la mayoría de las muestras estudiadas tienen un origen marino carbonático, correspondiente a la familia II de petróleos cubanos, este es el caso de las muestras LB1, AM2, SMB, MO, ME, LR y CC. Las muestras FS, CB y M muestran características de ambas familias (II y III), quedando ubicadas después de un minucioso análisis de sus características geoquímicas y del análisis estadístico, la muestra de FS como perteneciente a la familia II y las muestras de CB y M como pertenecientes a la familia III de petróleos cubanos depositadas en ambiente marino fundamentalmente en rocas marl-siliciclásticas con mayor aporte bacterial que algal-terrígeno, depositadas en un ambiente subóxico de baja salinidad.

Todas las muestras estudiadas están afectadas por biodegradación que alcanza hasta los esteranos en la muestra correspondiente a M.

La muestra de CB se presenta como la de mayor evolución térmica, según el conjunto de parámetros estudiados.

\section{MATERIAL SUPLEMANTAR}

Figuras $1 \mathrm{~S}$ a $3 \mathrm{~S}$ estan diponibilizadas gratuitamente en la pagina web http://quiicanova.sbq.org.br en la forma de un archivo .PDF.

\section{AGRADECIMIENTOS}

$\mathrm{Al}$ CNPq por las becas; a la Petrobras, en especial al CENPES por el soporte laboratorial.

\section{REFERENCIAS}

1. López, J. O.; Pascual, O.; Delgado, O.; López, J. G.; Resumen extendido IX Congreso Latinoamericano de Geoquímica Orgánica de ALAGO, Mérida, México, 2004; López, J. O.; Pascual, O., López, J. G.; Delgado, O.; Laffita, M. C.; Resumen extendido X Latinamerican Congress on Organic Geochemistry, Salvador, Brasil, 2006; Pascual, O.; López, J. O.; Delgado, O.; López, J. G.; Resumen extendido X Latinamerican Congress on Organic Geochemistry, Salvador, Brasil, 2006; Domínguez, Z.; López, J. O.; Guzzo, J. V. P.; Azevedo, D. A.; III Congresso Brasileiro de P\&D em Petróleo e Gás, Salvador, Brasil, 2005; Domínguez, Z.; López, J. O.; Guzzo, J. V. P.; Azevedo, D. A.; Resumen extendido X Latinamerican Congress on Organic Geochemistry, Salvador, Brasil, 2006.

2. López, O.; Tesis de Maestria, Instituto Superior Politécnico José Antonio Echevarria, Cuba, 2003.

3. Alexander, R.; Larcher, A. V.; Kagi, R. I.; Price, P. L. En Biological Markers in Sediments and Petroleum; Moldowan, J. M.; Albrecht, P.; Philp, R. P., eds.; Prentice-Hall: Englewood Cliffs, 1992.

4. Peters, K. E.; Moldowan, J. M.; The Biomarker Guide; Interpreting Molecular Fossils in Petroleum and Ancient Sediment, Eglewood Cliffs, Prentice Hall: New York, 1993; Peters, K. E.; Walters, C. C.; Moldowan, J. M.; The Biomarker Guide: Biomarkers and Isotopes in the Petroleum Exploration and Earth History, $2^{\text {nd }}$ ed., Cambridge University Press: New York, 2005, vol. 12.

5. Aquino Neto, F. R.; Trendel, J. M.; Restlé, A.; Connan, J.; Albrecht, P. En Advances in Organic Geochemistry; Bjoroy, M.; Albrecht, P.; Cornford., C., eds.; John Wiley \& Sons: New York, 1983.

6. Seifert, W. K.; Moldowan, S. M.; Geochim. Cosmochim. Acta 1979, 43, 111; Palacas, J. G.; Monopolis, D.; Nicolau, C. A.; Anders, D. E.; Org. Geochem. 1986, 10, 417. 
7. Alexander, R.; Kagi, R. J.; Rowland, S. J.; Sheppard, P. N.; Chirila, T. V.; Geochim. Cosmochim. Acta 1985, 49: 385; Ellis, L.; Langworthy, T. A.; Winans, R.; Org. Geochem. 1996, 24, 57; Fan, P.; Philp, R. P. Lizhenxi, Y. X.; Ying, G.; Chem. Geol. 1991, 93: 61; Farrington, J. W.; Davis, A. C.; Tarafa, M. E.; Org. Geochem. 1988, 13, 303; Mackenzie, A. S.; Hoffmann, C. F.; Maxwell, J. R.; Geochim. Cosmochim. Actc 1981, 45, 1345; Mackenzie, A. S.; Lamb, N. A.; Maxwell, J. R.; Nature 1982, 295, 223; Moldowan, J. M.; Fago, F. J.; Geochim. Cosmochim. Acta 1986, 50, 343; Payzant, J. D.; Montgomery, D. S.; Strausz, O. P.; Tetrahedron Lett. 1983, 24, 651; Radke, M.; Welte, D. H.; Willsch, H.; Geochim. Cosmochim. Acta 1982a, 46, 1; Radke, M.; Mar. Pet. Geol. 1988, 5, 224; Shi, J-Y.; Mackenzie, A. S.; Alexander, R.; Eglinton, G.; Gowar, A. P.; Wolff; G. A.; Maxwell, J. R.; Chem. Geol. 1982, 35, 1.

8. Marzi, R.; Rullkotter, J. En Biological Markers in Sediment and Petroleum; Moldowan, J. M., ed.; Prentice Hall: Englewood Cliffs, 1992.

9. Mackenzie, A. S. En Advances in Petroleum Geochemistry; Brooks, C. J. W.; Welte, D. H., eds.; Academic Press: London, 1984.

10. Radke, M. En ref. 9.

11. Radke, M.; Welte, D. H.; Willisch, H.; Org. Geochem. 1986, 10, 51 ; Boreham, . J.; Crick, I. H.; Powell, T.G.; Org. Geochem. 1988, 12, 289.
12. Hugues, W.B. En Petroleum Geochemistry and Source Rock Potencial of Carbonate Rock; Palacas, J. G., ed.; J. B. AAPG Studies in Geology, $18,1984$.

13. Schou, L.; Myhr, M. B.; Org. Geochem. 1988, 13, 61.

14. Volkman, J. K.; Alexander, R.; Kagi, R. I.; Rowland, S. F.; Shepard, P. N.; Org. Geochem. 1984 6, 619; Williams, J. A.; Bjoroy, M.; Dolcater, D. L.; Winters, J. C.; Org. Geochem. 1986, 10, 451.

15. Cassani, F.; Eglinton, G.; Chem. Geol. 1985, 56, 167.

16. Rullkotter, J.; Spiro, B.; Nissenbaum, A.; Geochim. Cosmochim. Acta 1985, 49, 1357

17. Rullköter, J.; Marzi, R.; Org. Geochem. 1988, 13, 639.

18. Hugues, W. B.; Holba, A. G.; Dzou, L. I.; Geochim. Cosmochim. Acta 1995, 59, 3581

19. Chakmakhchev, A.; Suzuki, N.; Takayama, K.; Org. Geochem. 1997, 26, 483.

20. Spiegel, M.; Estadística, McGraw-Hill: Madrid, 1991; Guimarães, R. C.; Cabral, J.; Estatística, McGraw-Hill: Lisboa, 1997; Johnson, R.; Wichern, D.; Applied Multivariate Statistical Analysis, Prentice-Hall: Englewood Cliffs, 1998.

21. Karypis, G.; Han, E. H. S.; News, V. K.; Comput. 1999, 32, 68.

22. Everitt, B. S.; Cluster Analysis, Hodder \& Stoughton: London, 1993. 


\section{CARACTERIZACIÓN Y CLASIFICACIÓN GEOQUÍMICA DE ASFALTITAS CUBANAS}

\section{Zulema Dominguez}

COPPE/UFRJ - Instituto Alberto Luiz Coimbra de Pós-graduação e Pesquisa em Engenharia, Universidade Federal de Rio de Janeiro, Rio de Janeiro - RJ, Brasil / CEINPET/CUPET -Centro de Investigaciones del Petróleo, Habana, Cuba

Jarbas Vicente Poley Guzzo

Petrobras/CENPES/PDEXP/GEOQ, Ilha do Fundão, 21941-901 Rio de Janeiro - RJ, Brasil

Débora de Almeida Azevedo*

Instituto de Química, Universidade Federal de Rio de Janeiro, 21941-909 Rio de Janeiro - RJ, Brasil

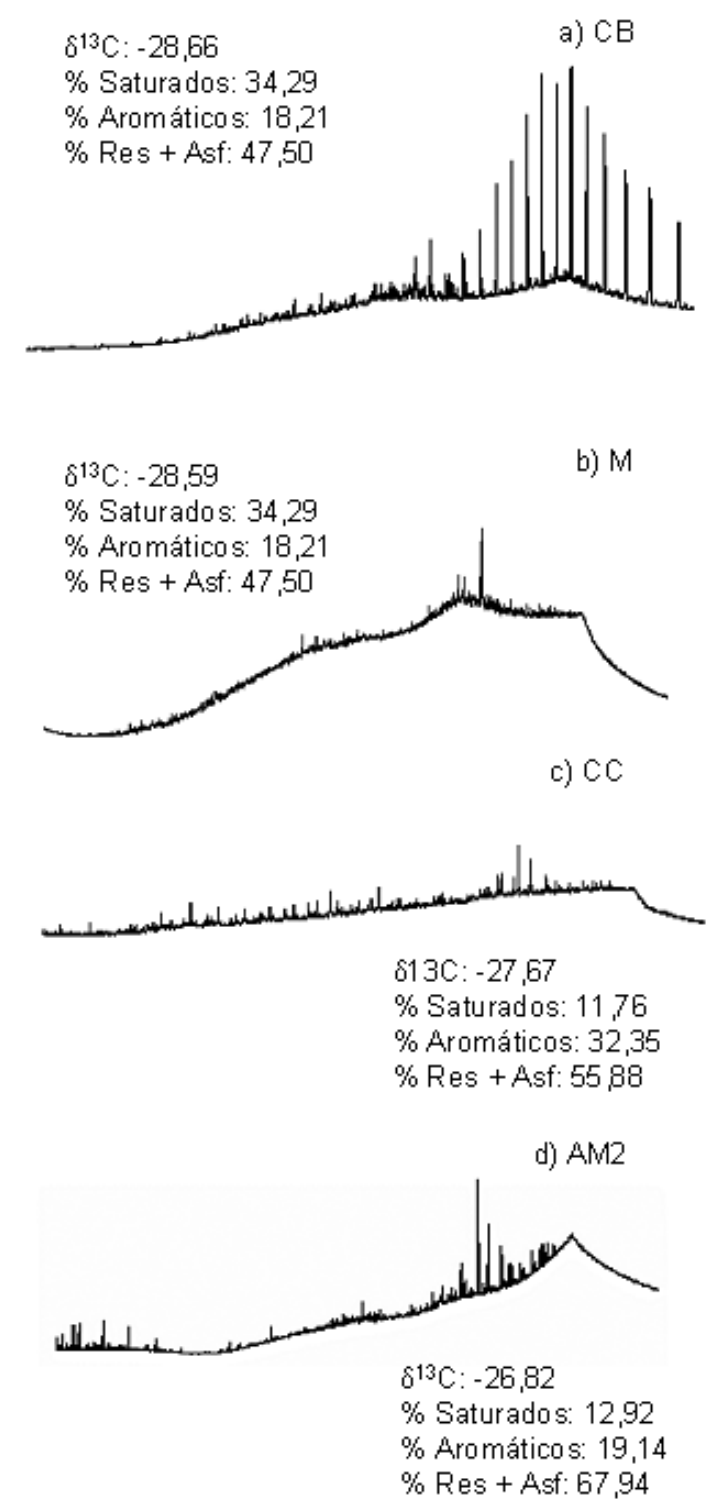

Figura 1S. Corresponde a los cromatogramas de gases de la fracción saturada 
a) CB

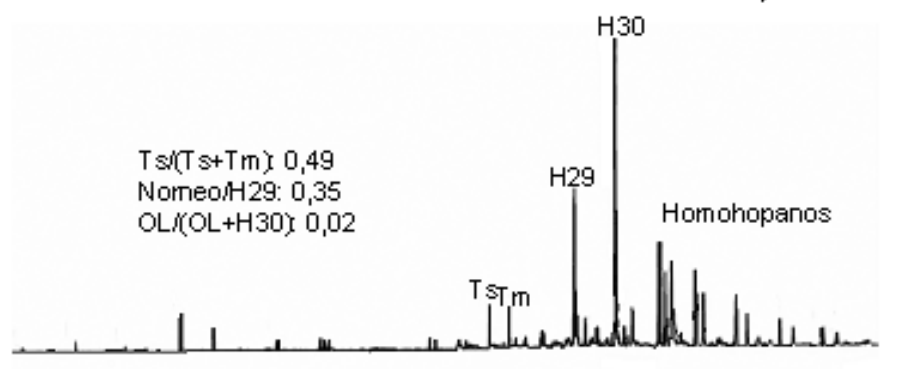

$20 S /(20 S+20 R)$ St: 0,55

$\alpha \alpha \alpha j(\alpha \beta \beta+\alpha \alpha \alpha): 0,46$

$\% 27 \alpha \beta \beta R \& S$ (218): 27,41

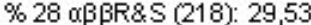

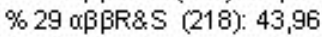

DIARE G Colestanos: 0,34
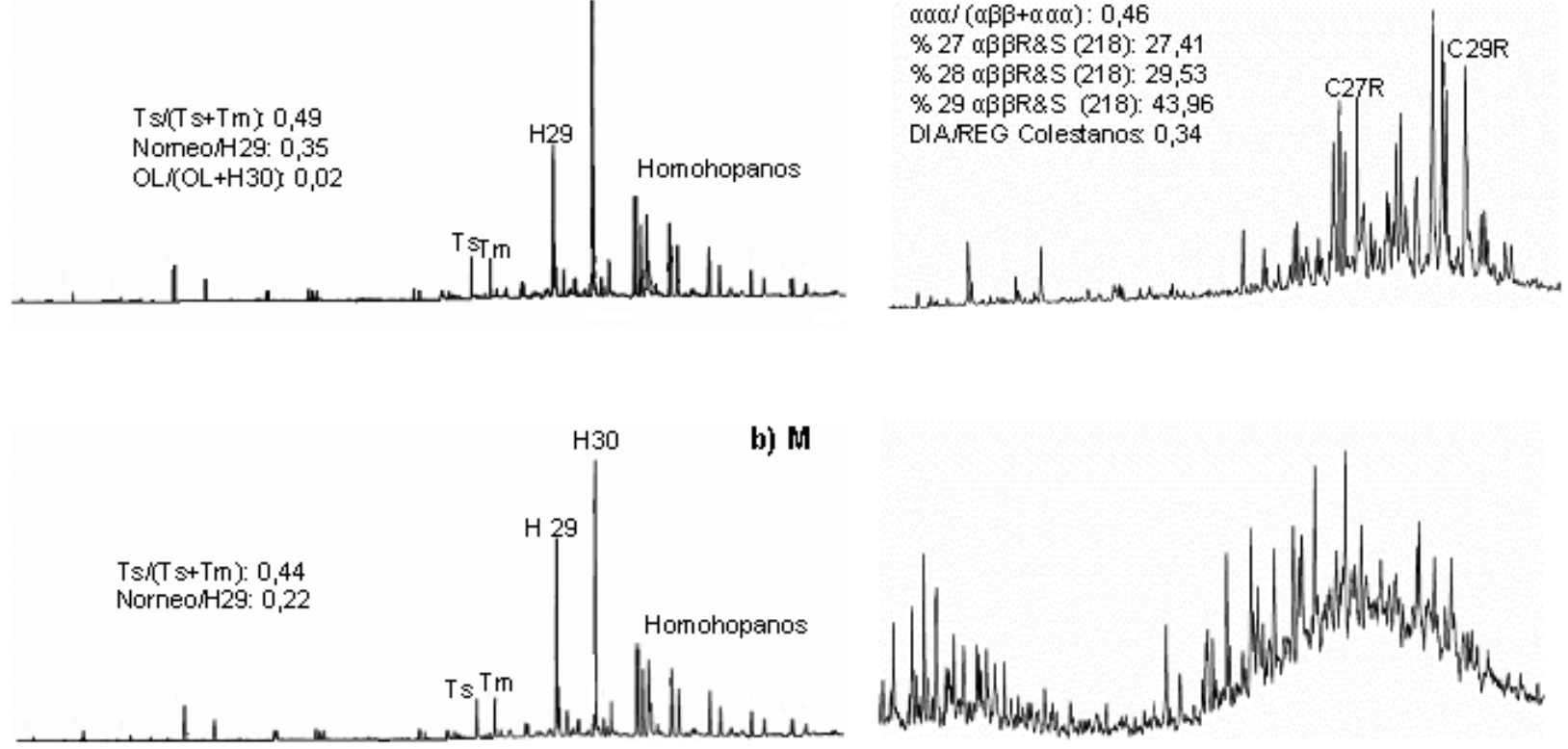

c) $\mathrm{CC}$
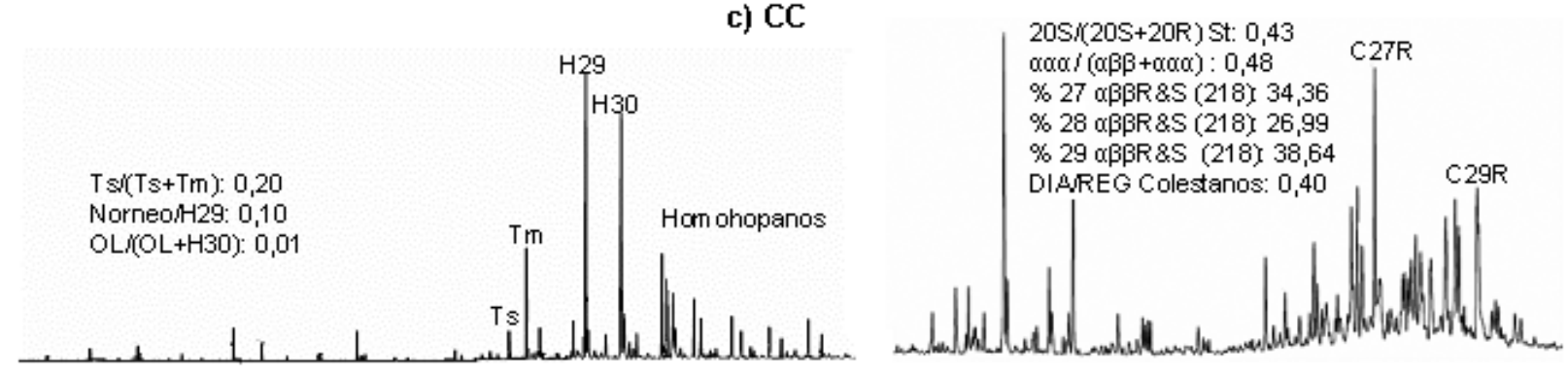

d) $\mathrm{Al} 2$
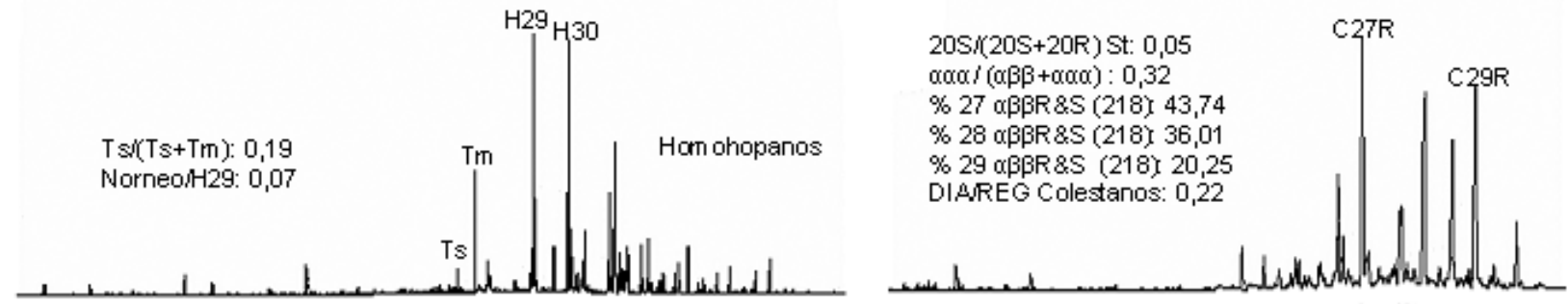

Figura 2S. Ejemplos más representativos de los cromatogramas de masas de biomarcadores saturados (m/z 191 y m/z 217): Tm es C27 17 $\alpha(\mathrm{H})$-22,29,30-tris-

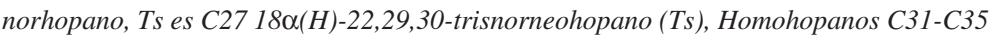


a) CB

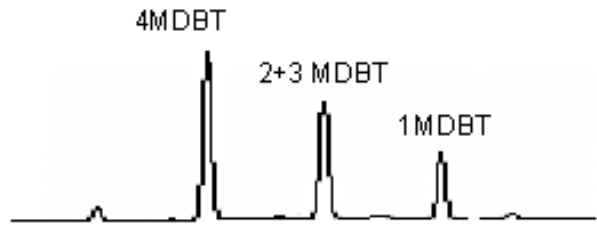

b) $M$
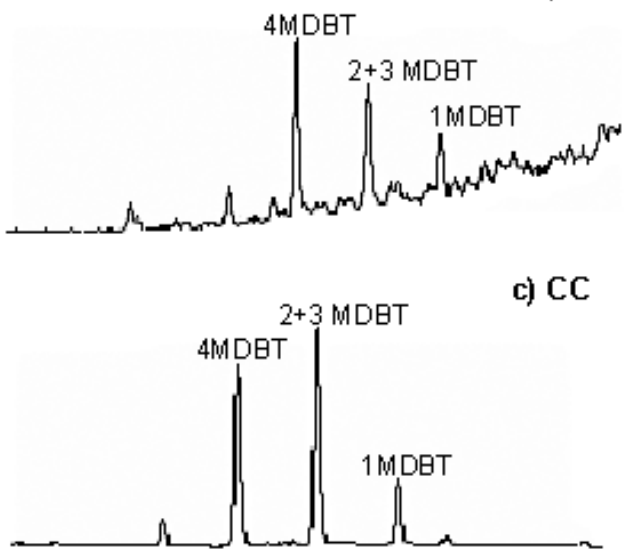

d) AM2 2

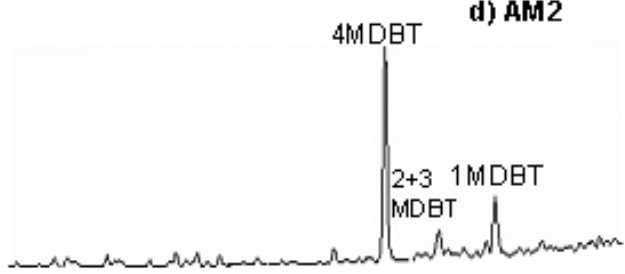

Figura 3S. Ejemplos más significativos de los cromatogramas de masas de metildibenzotiofenos (m/z 198) 